\title{
Gastric carcinogenesis in the rat induced by duodenogastric reflux without carcinogens: morphology, mucin histochemistry, polyamine metabolism, and labelling index
}

\author{
P R Taylor, R C Mason, M I Filipe, S Vaja, D C Hanley, G M Murphy, R H Dowling, I McColl
}

\begin{abstract}
Chronic duodenogastric reflux induces gastric adenocarcinomas in the rat without the use of carcinogens. Altogether, 186 male Wistar rats were randomised to undergo either a simple gastrojejunostomy or a gastrotomy and sacrificed at eight weekly intervals for 56 weeks. No control animals developed dysplasia or carcinoma. All rats subjected to a gastrojejunostomy showed hyperplasia of the proliferative neck zone, with increased sulphomucin production adjacent to the scar. Low grade dysplasia was found at 16 weeks, and carcinoma was first seen at 32 weeks. Most carcinomas were well differentiated mucin secreting adenocarcinomas of the expanding type, which secreted a mixture of sialomucins and sulphomucins. Duodenogastric reflux was associated with a $100 \%$ increase in labelling index (assessed autoradiographically with tritiated thymidine) in the gastric mucosa when compared with corresponding tissue adjacent to a gastrotomy scar. This increase was significant at eight weeks and persisted for 56 weeks after surgery. This study supports the theory that, in this model, hyperplasia precedes the development of carcinoma.
\end{abstract}

The incidence of gastric stump cancer remains controversial. ${ }^{1-6} \mathrm{~A}$ large recent series, however, has shown that gastric surgery for benign disease is associated with a pronounced increase in gastric adenocarcinoma when compared with a control population from the same locality.

Studies in experimental animals have shown that gastric surgery can induce adenocarcinoma of the stomach without the use of carcinogens. ${ }^{8}$ Furthermore, the incidence of malignant change in the gastric mucosa was proportional to the degree of duodenogastric reflux. In this rat model of duodenogastric reflux the pancreaticoduodenal secretions, rather than the bile, seemed to be responsible for the tumour. ${ }^{9}$ In tissues which have a high proliferative rate the activity of the rate-limiting enzyme in polyamine biosynthesis, ornithine decarboxylase, and the concentrations of the polyamines are relatively high. Many human cancers also show high levels of ornithine decarboxylase activity in the malignancy. ${ }^{11}$ Since duodenogastric reflux is a reliable model for inducing gastric cancer, and since polyamine metabolism has never been studied in the gastric mucosa in this model, we examined the sequential longterm effects of duodenogastric reflux on gastric mucosal morphology, mucin histochemistry, ornithine decarboxylase activity, and polyamine concentration and correlated the results with the labelling index assessed autoradiographically, an established method of measuring tissue proliferation.

\section{Methods}

STUDY DESIGN

Altogether, 186 male Wistar rats weighing 200 $250 \mathrm{~g}$ were randomised to undergo either simple gastrojejunostomy or gastrotomy. Laparotomy was performed through an upper midline abdominal incision and a $7 \mathrm{~mm}$ gastrotomy was made $2 \mathrm{~mm}$ distal to the squamocolumnar junction along the anterior surface of the greater curvature. In gastrotomy rats this was closed with an all layer 6/0 Ethibond suture. In gastrojejunostomy animals a loop of jejunum $4 \mathrm{~cm}$ distal to the ligament of Treitz was anastomosed to the stomach using an all layer 6/0 Ethibond suture in an isoperistaltic, antecolic manner. Animals from the two groups which survived the operation (operative mortality $10.8 \%$ ) were allocated to cohorts to be sacrificed at $8,16,24$, $32,40,48$, and 56 weeks after surgery. An intraperitoneal injection of tritiated thymidine (specific activity $5 \mathrm{Ci} / \mathrm{mmol}$, Amersham International) was given one hour before sacrifice at a dose of $1 \mu \mathrm{Ci} / \mathrm{g}$ body weight.

The rats were killed by exsanguination under general anaesthetic. The stomach was removed en bloc and opened along the greater curvature. After washing briefly in tap water, the stomach was pinned out on a cork board and the macroscopic findings noted. Some $20-70 \mathrm{mg}$ samples of gastric mucosa were removed by sharp dissection from the underlying serosa and muscle immediately adjacent to the gastrotomy or to the anastomosis. The samples were stored at $-70^{\circ} \mathrm{C}$ until analysed for polyamines and related enzymes within three months of sacrifice.

The stomach was fixed in $10 \%$ formol saline, processed in wax, and seven serial $3 \mu \mathrm{m}$ sections of each block were prepared for morphological assessment with haematoxylin and eosin, and with alcian blue - periodic acid-Schiff and, high iron diamine-alcian blue to distinguish neutral, sialomucins, and sulphomucins.'

Two $1 \mu \mathrm{m}$ sections from each block were prepared for autoradiography using the dipping method with $\mathrm{K} 5$ emulsion (Ilford). ${ }^{13} \mathrm{~A}$ minimum of 500 cells in each part of the mucosa under study was counted and the number of labelled 
cells noted. The labelling index was the number of labelled cells per 100 cells counted. With each batch a section from a control animal which had not been injected with tritiated thymidine was used to assess background activity .

\section{HISTOLOGICAL CLASSIFICATION}

Histological lesions were classified into the following groups: atrophy, normal mucosa found in deeper layers, downward glandular growth, intestinal metaplasia, hyperplasia, adenocystic glandular proliferation, reactive atypia, dysplasia (low and high grade), and carcinoma.

Atrophy of the mucosa was associated with a decrease in mucosal depth and could affect either the antrum or the body mucosa. Normal epithelium of either gastric or small bowel was often found in deeper layers due to the disruption caused by surgery. They were readily identified by normal histology and mucin pattern. Downward glandular growth referred to benign appearing glands found beneath the muscularis mucosa at the anastomosis with no evidence of atypia. Hyperplastic mucosa was characterised by lengthening of the foveolae and neck regions. Adenocystic glandular proliferation was a serosally orientated growth of an increased number of glands which were cystically dilated and devoid of atypical features. Intestinal metaplasia was diagnosed by the finding of goblet cells within the gastric mucosa. The abnormal mucosa was separated from the small bowel mucosa at the anastomosis by a variable portion of gastric mucosa so that transposition of small bowel mucosa by surgery was unlikely.

Reactive atypia showed a disorganised architecture with a slight increase in the number of mitoses adjacent to an area of erosion or ulceration.

Polyps were classified into three types. Hyperplastic polyps showed an elongation and increase in number of glands which were disorganised and often associated with foveolar hyperplasia. Muscle fibres were seen within them but there was no inflammation. Inflammatory or reactive polyps were usually adjacent to areas of ulcera-

TABLE I Histological findings in the gastrotomy $(G)$ and gastrojejunostomy $(G \mathcal{f})$ groups

\begin{tabular}{|c|c|c|c|c|c|c|c|c|}
\hline & \multicolumn{8}{|l|}{ Weeks } \\
\hline & $\stackrel{8}{G / G \mathcal{F}}$ & $\begin{array}{l}16 \\
G / G f\end{array}$ & 24 & $\begin{array}{l}32 \\
G / G \mathcal{f}\end{array}$ & $\stackrel{40}{G / G f}$ & $\stackrel{48}{G / G \mathcal{f}}$ & $\begin{array}{l}56 \\
G / G \mathcal{f}\end{array}$ & $\begin{array}{l}\text { Total } \\
\text { G/Gf }\end{array}$ \\
\hline No & $12 / 12$ & $12 / 12$ & $13 / 12$ & $12 / 10$ & $12 / 12$ & $12 / 13$ & $12 / 13$ & $85 / 84$ \\
\hline Loss of specialised cells & $12 / 12$ & $12 / 12$ & $13 / 12$ & $11 / 10$ & $12 / 12$ & $12 / 13$ & $12 / 13$ & $84 / 84$ \\
\hline Foveolar hyperplasia & $12 / 12$ & $12 / 12$ & $13 / 12$ & $11 / 10$ & $12 / 12$ & $12 / 13$ & $12 / 13$ & $84 / 84$ \\
\hline Neck hyperplasia & $6 / 12$ & $-/ 12$ & $3 / 12$ & $1 / 9$ & $3 / 10$ & $-/ 13$ & $1 / 13$ & $14 / 81$ \\
\hline Downward glandular proliferation & $5 / 2$ & $7 / 10$ & $6 / 8$ & $7 / 4$ & $7 / 10$ & $6 / 5$ & $4 / 5$ & $42 / 44$ \\
\hline Normal epithelium in deeper layers & $2 / 1$ & $6 / 8$ & $7 / 7$ & $2 / 7$ & $4 / 2$ & $2 / 2$ & $1 / 1$ & $24 / 28$ \\
\hline Adenocystic glandular proliferation & $-1-$ & $1 / 3$ & $1 / 1$ & $2 / 2$ & $4 / 4$ & $7 / 6$ & $7 / 6$ & $22 / 22$ \\
\hline Bone metaplasia & $1 /-$ & -12 & $1 / 2$ & $2 / 4$ & -16 & $5 / 6$ & $2 / 5$ & $11 / 25$ \\
\hline Antral atrophy & -16 & -18 & -18 & $-/ 8$ & $-/ 8$ & -19 & $-/ 11$ & -158 \\
\hline Inflammatory polyp & $-1-$ & $-1-$ & $-/ 1$ & $1 / 1$ & $1 / 3$ & $3 / 2$ & -13 & $5 / 10$ \\
\hline Hyperplastic polyp & $0 / 1$ & $0 /-$ & $0 /-$ & $0 /-$ & $0 / 1$ & $0 / 1$ & $0 /-$ & $0 / 3$ \\
\hline Adenomatous polyp & $0 /-$ & $0 /-$ & $0 /-$ & $0 /-$ & $0 / 1$ & $0 /-$ & $0 /-$ & $0 / 1$ \\
\hline Reactive atypia & $1 / 3$ & $2 / 3$ & -15 & $1 / 4$ & -14 & $1 / 1$ & $1 / 1$ & $6 / 21$ \\
\hline Low grade dysplasia & $-1-$ & $-/ 1$ & $-/ 1$ & $-1-$ & $-/ 1$ & $-/ 1$ & $-/ 1$ & -15 \\
\hline High grade dysplasia & $-1-$ & $-1-$ & $-1-$ & $-1-$ & $-1-$ & $-/ 1$ & $-1-$ & $-/ 1$ \\
\hline Intramucosal carcinoma & $-1-$ & $-1-$ & $-1-$ & $-1-$ & $-/ 1$ & $-/ 1$ & $-1-$ & -12 \\
\hline Advanced carcinoma & $-1-$ & $-1-$ & $-1-$ & $-/ 1$ & $-1-$ & $-/ 1$ & $-/ 2$ & -14 \\
\hline Paneth cell metaplasia & $1 / 6$ & $3 /-$ & -14 & -12 & $1 / 1$ & $1 / 1$ & $-/ 1$ & $6 / 15$ \\
\hline Intestinal metaplasia & $-/ 1$ & -12 & $-1-$ & -12 & $-1-$ & -13 & $-/ 1$ & -19 \\
\hline $\begin{array}{l}\text { Hyperplasia of all elements of the } \\
\text { body mucosa }\end{array}$ & $3 / 11$ & $-/ 11$ & -19 & -13 & $-/ 11$ & $6 / 11$ & -19 & $9 / 65$ \\
\hline Ulcer/erosion & $1 / 4$ & -15 & -19 & -13 & $1 / 8$ & -16 & $-/ 6$ & 2/41 \\
\hline
\end{tabular}

tion with granulation tissue. Adenomatous polyps showed disorganised architecture with degrees of cellular immaturity. The glands showed abnormal differentiation with an increased number of mitoses.

Dysplastic glands usually had a reduced mucin secretion in an area of disorganised architecture with an increase in nuclear pleomorphism and number of mitoses. These were classified into low and high grades depending on the severity of the changes.

Carcinomas were characterised by pronounced nuclear pleomorphism with increased mitotic figures, bizarre and severely disorganised architecture, and invasion beyond the muscularis mucosa.

\section{LABORATORY ASSAYS}

\section{Ornithine decarboxylase activity}

Ornithine decarboxylase activity was measured using the method of Luk and Baylin. ${ }^{14}$ Liquid scintillation spectroscopy was used to detect radiolabelled carbon dioxide liberated by the decarboxylation of ornithine by ornithine decarboxylase. A blank was prepared with each batch to assess background scintillation activity. Difluoromethylornithine, a specific irreversible inhibitor of ornithine decarboxylase, was added to some samples to show whether the activity being measured by the assay was specific ornithine decarboxylase activity.

\section{Protein}

Protein assay was performed by a colorimetric method using alkaline copper reagent and

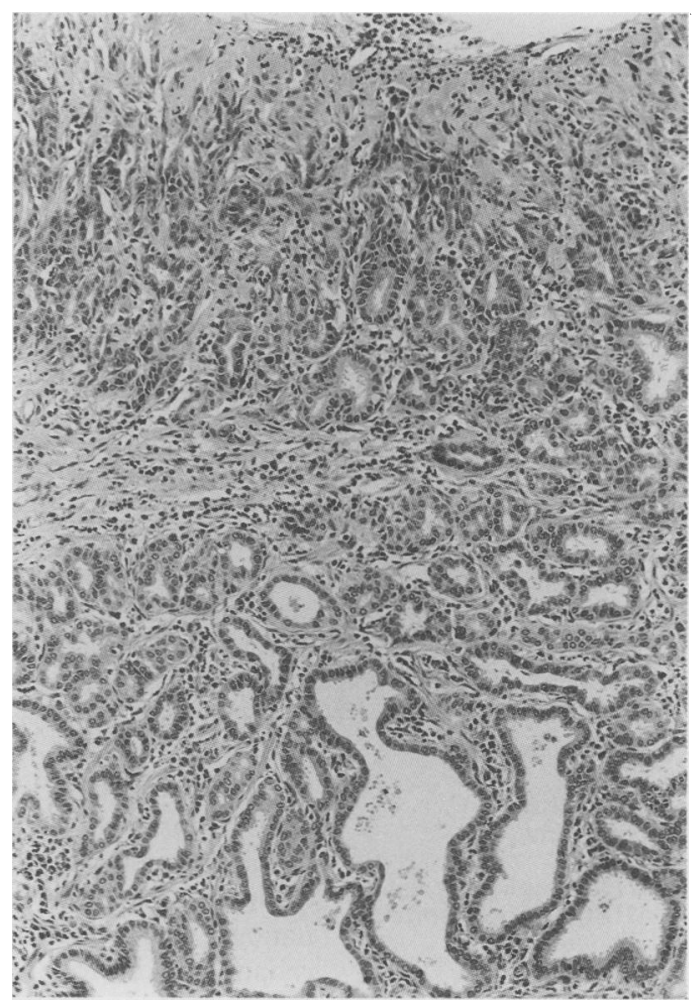

Figure 1: Area of adenocystic glandular proliferation in the submucosa consisting of large spaces lined by benign appearing cells. There is an erosion of the overlying mucosa. Haematoxylin $\mathcal{E}$ eosin, original magnification $\times 175$. 
Figure 2: Atrophic mucosa with extensive bone metaplasia (arrowed) and adenocystic glandular proliferation. Haematoxylin $\mathcal{E}$ eosin, original magnification $\times 70$.

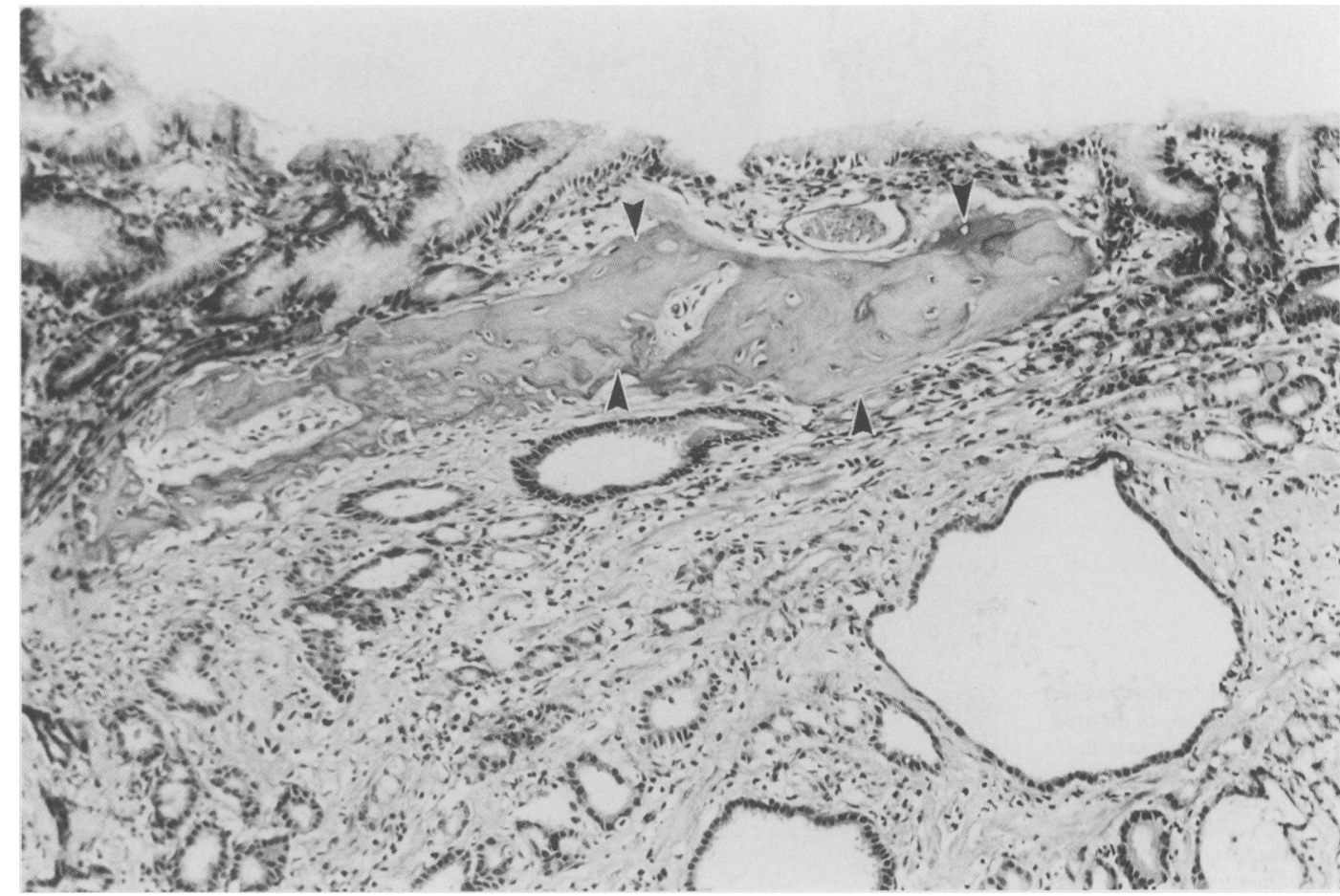

Folin's and Ciocalteu's reagent. ${ }^{15}$ The samples were read at $750 \mathrm{~nm}$ and compared with standards made from bovine serum albumin.

\section{Polyamines}

A high performance liquid chromatographic procedure (described by Sieler and Knodgen) was used to analyse the gastric mucosal polyamines. ${ }^{16}$ An internal standard (1,7 diaminoheptane dihydrochloride) was used with each run and a standard mixture of polyamines was used after every eight samples to control instrument sensitivity and reproducibility. Results are given for putrescine and spermine and for total spermidine (the sum of non-acetylated spermidine and the N1 and N8 acetylated derivatives).

\section{STATISTICAL METHOD}

The significance of the differences in the results of polyamine concentrations and ornithine decarboxylase activity was determined by the Mann-Whitney $U$ test. Histological findings were assessed by $\chi^{2}$ analysis with Yates's correction factor for small numbers, and by Fisher's exact test where appropriate. If the $p$ value was less than 0.05 the results were considered significant.

\section{Results}

MORPHOLOGY AND MUCIN HISTOCHEMISTRY

The results are shown in Table I. Eighty four animals with a gastrojejunostomy were compared with 85 rats with a gastrotomy. All animals showed pseudopyloric metaplasia adjacent to the scar with atrophy of the mucosa, loss of specialised cells, an increase in sialomucins in the remaining glands, and foveolar hyperplasia containing neutral mucins. In contrast to the controls, 81 of 84 gastrojejunostomy animals also showed pronounced hyperplasia of the proliferative neck zone, associated with increased sulphomucin production adjacent to the scar $(\mathrm{p}<0.001)$. This was a constant finding throughout the experimental period.

Features which did not show a significant difference in incidence to control rats included normal epithelium taken into deeper layers, downward glandular proliferation, and adenocystic glandular proliferation (Fig 1). During the experiment the number of animals with normal epithelium in deeper layers decreased whereas the number with adenocystic glandular proliferation increased. This relation was also found in gastrotomy rats. Fifty eight gastrojejunostomy animals had atrophy (Fig 2) of the antral mucosa $(p<0.001)$ and 65 showed hyperplasia of all elements of the body mucosa next to the area of pseudopyloric metaplasia $(\mathrm{p}<0.001)$.

Forty one rats with a gastrojejunostomy had erosions or ulceration of either the gastric or the jejunal mucosa $(\mathrm{p}<0.001)$. The jejunum frequently showed the features of villus atrophy and crypt cell hyperplasia.

Macroscopic polyps were much larger and more numerous $(p<0.05)$ in the gastrojejunostomy group and microscopically these were inflammatory, hyperplastic, or adenomatous. Only inflammatory polyps were found in the control animals. All polyps were closely associated with suture material.

Bone metaplasia was twice as common in gastrojejunostomy rats $(p<0.01)$ and increased with time. Fifteen animals had Paneth cell metaplasia $(p<0.05)$, but in contrast to the controls, nine animals showed focal areas of intestinal metaplasia $(\mathrm{p}<0.001)$.

Reactive atypia (Fig 3) was found throughout the study in 21 gastrojejunostomy animals and decreased with time $(p<0 \cdot 01)$.

No gastrotomy animals developed dysplasia or carcinoma. In the gastrojejunostomy group, low grade dysplasia was found $(\mathrm{p}<0.02)$ at 16 weeks 
Figure 3: The mucosa shows reactive changes, in an area of erosion and ulceration, with disorganised glandular architecture and regular epithelium. Haematoxylin E' eosin, original magnification $\times 175$.

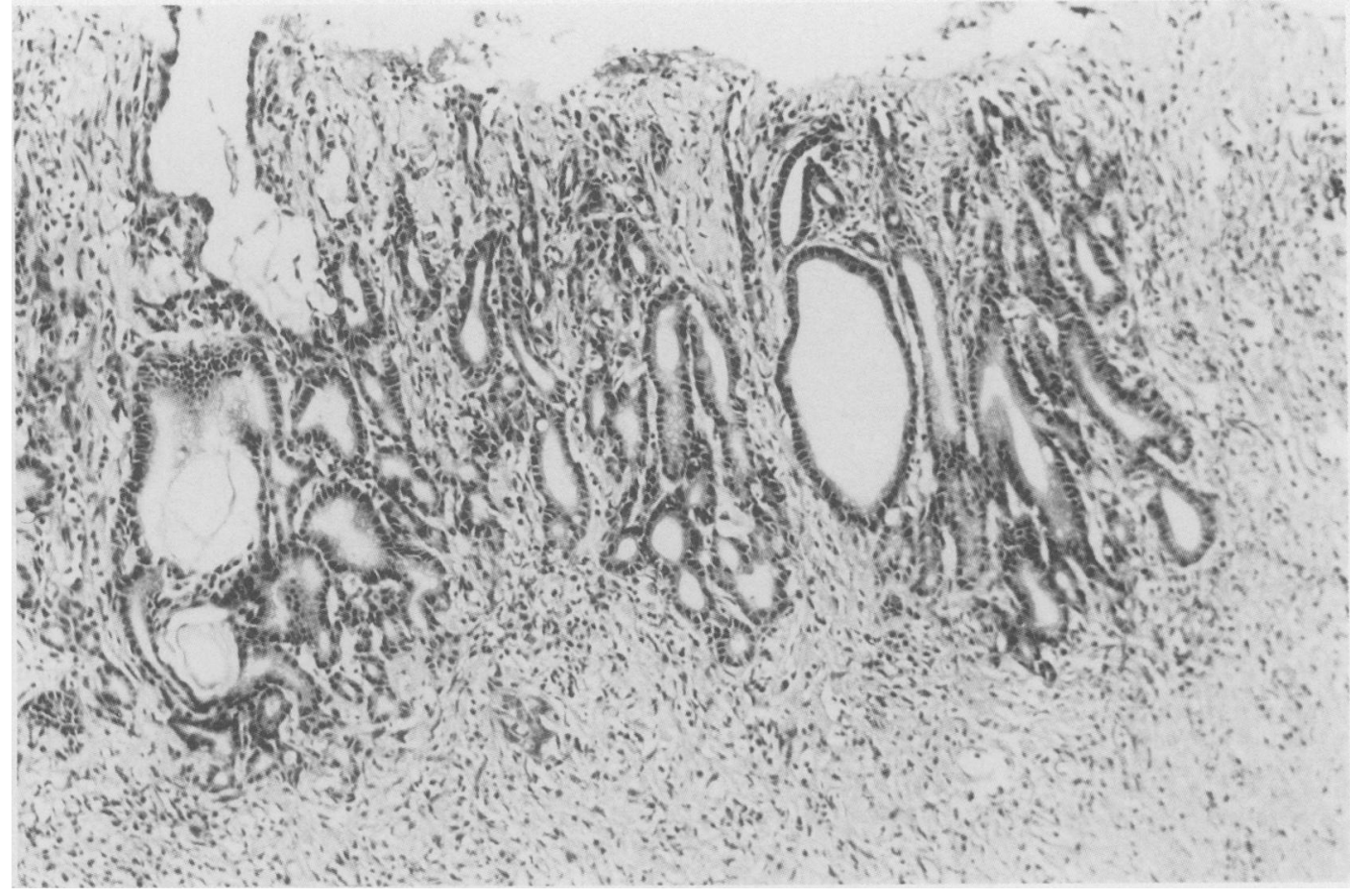

and occurred in one animal in each group thereafter throughout the experiment with the exception of the 32 week cohort. In one case this was associated with intestinal metaplasia (Fig 4).

High grade dysplasia was found in one animal at 48 weeks in an area of adenocystic glandular proliferation. Both grades of dysplasia were associated with a decrease in mucin production. Intramucosal carcinoma was found in a flat mucosa associated with intestinal metaplasia in one animal at 40 weeks and in a large adenomatous polyp at 48 weeks. Four carcinomas were diagnosed: one each at 32 and 48 weeks and two at 56 weeks. Three of these were serosally directed growths consisting of well differentiated, mucin secreting adenocarcinomas of the expanding variety of Lauren's classification (Fig 5). All of these had breached the serosa. The remaining one was a luminally directed polypoid adenocarcinoma found at 56 weeks. Carcinomas secreted a mixture of sialomucins and sulphomucins. No lymph node metastases or distant metastases were found. High grade dysplasia and carcinomas only occurred in the gastrojejunostomy group $(\mathrm{p}<0.006)$.

\section{ORNITHINE DECARBOXYLASE ACTIVITY}

The results for combined values for ornithine decarboxylase activity together with those of the polyamines are shown in Figure 6. The ornithine decarboxylase results, subdivided into various time intervals after surgery, are shown in Figure 7. Activity was assessed in almost all animals, and polyamine concentration and labelling index in $50 \%$ of the animals in each group.

The pooled results, independent of time after surgery, showed that the mean ornithine decarboxylase activity was $36 \%$ higher in the gastrojejunostomy group when compared with the gastrotomy control. This pattern of results was also true at every time interval after surgery, with $13-72 \%$ increases of enzyme activity in the gastrojejunostomy group when compared with the gastrotomy controls. These differences were significant only at 16,48 , and 56 weeks, however. Difluoromethylornithine completely abolished ornithine decarboxylase activity, suggesting that the activity measured by the assay was specific to the ornithine decarboxylase enzyme.

POLYAMINES

The results for putrescine concentrations at

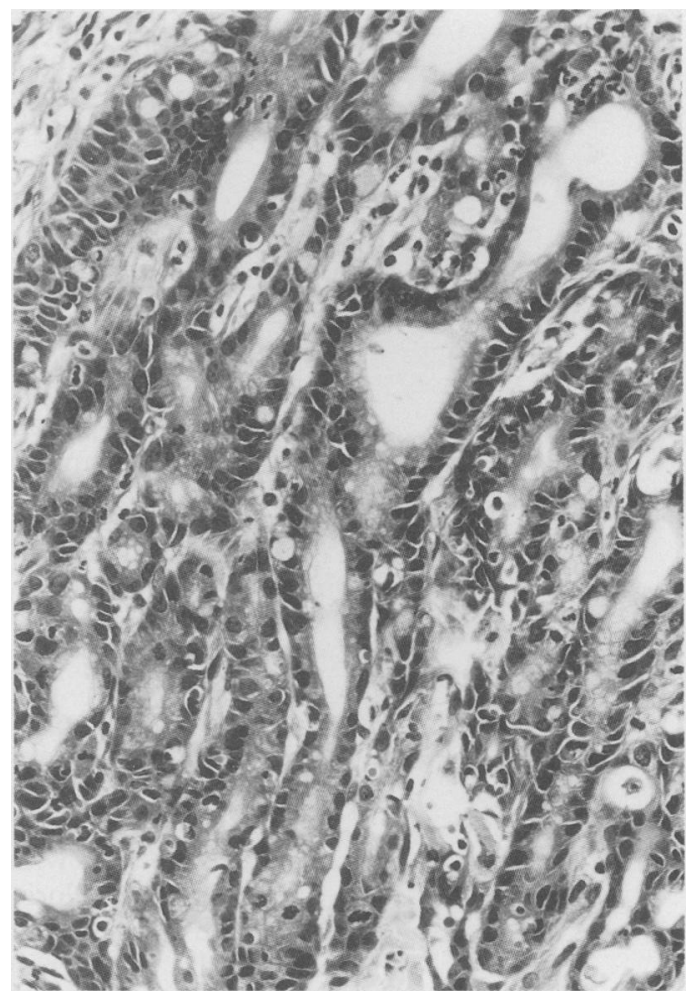

Figure 4: An area of intestinal metaplasia associated with low grade dysplasia. Haematoxylin $\mathcal{E}$ eosin, original magnification $\times 350$. 
Figure 5: Tubular mucus secreting adenocarcinoma. Haematoxylin $\mathcal{E}$ eosin, original magnification $\times 175$.
Figure 6: Combined values for ornithine decarboxylase activity and polyamine concentrations (mean $(S E M)) .{ }^{\star} p<0.05$ $\neq p<0 \cdot 01 ; \neq p<0 \cdot 001$

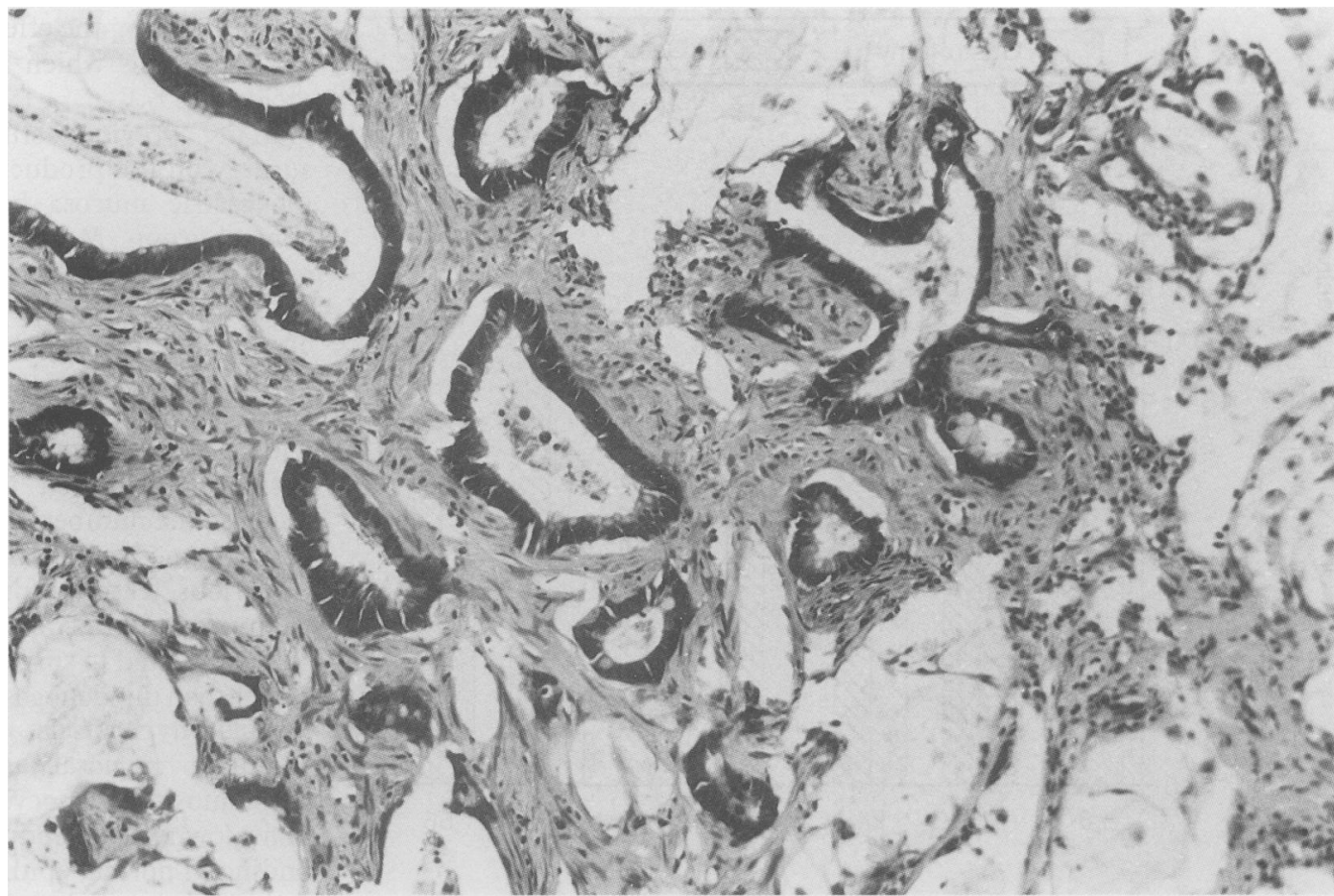

various time intervals after surgery are shown in Figure 8. Putrescine concentrations in the gastric mucosa were significantly increased by $44 \%$ in rats undergoing gastrojejunostomy compared with gastrotomy (Fig 6). Significantly raised concentrations of putrescine were found at 24 and 48 weeks (Fig 8). The mean putrescine concentrations, however, did not change significantly during the first two time periods, but thereafter the overall pattern of results was similar to that described above for ornithine decarboxylase activity, with $37-119 \%$ increases in the gastrojejunostomy group compared with gastrotomy controls.

There was no significant difference in spermidine concentration between the two groups taken as a whole (Fig 6) or at any time interval. Spermine concentrations were significantly higher in gastrotomy rats (Fig 6) but there was no consistent pattern with time and there was no significant difference between the two groups at any time interval.

\section{LABELLING INDEX}

The labelling index was calculated for both groups and is shown in Figure 9. The results show that the labelling index is significantly increased in the foveolae adjacent to the site of surgery in the gastrojejunostomy group compared with the gastrotomy controls at each time interval after surgery. The values for histopathological lesions found exclusively in the gastrojejunostomy group are shown in Table II. Both advanced carcinoma and intramucosal carcinoma had similar values. Low grade dysplasia showed values in the same range as those of hyperplasia found adjacent to the anastomosis in gastro-
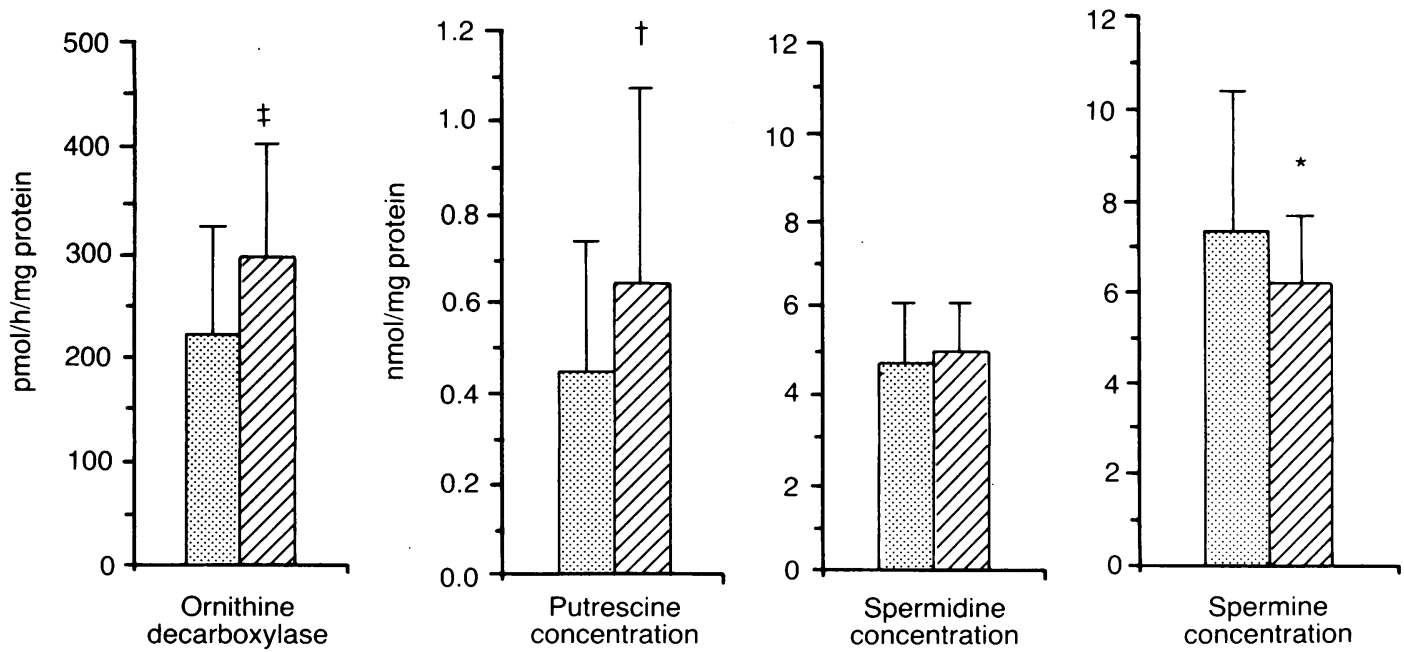
Gastrotomy Gastrojejunostomy

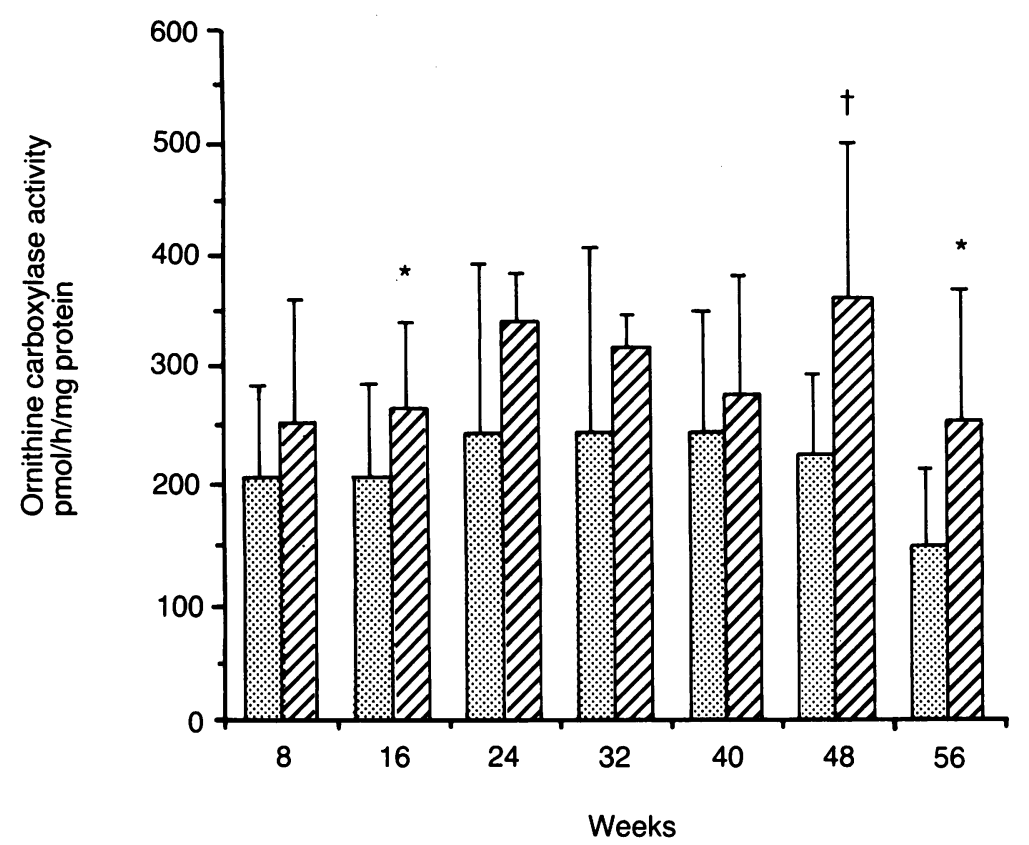

Figure 7: Ornithine carboxylase activity at various weeks after operation (mean (SEM)). $\star_{p}<0.05 ;+p<0.01$. jejunostomy animals. Polyps showed values intermediate between low grade and high grade dysplasia.

\section{Discussion}

The number of carcinomas found in this study was low compared with previous experiments ${ }^{89}$ and confirms the varying susceptibility of different strains of the Wistar rat to tumour induction. The incidence, however, of $15 \%$ found at 48 and 56 weeks is comparable with a recently published

\section{Gastrotomy Gastrojejunostomy}

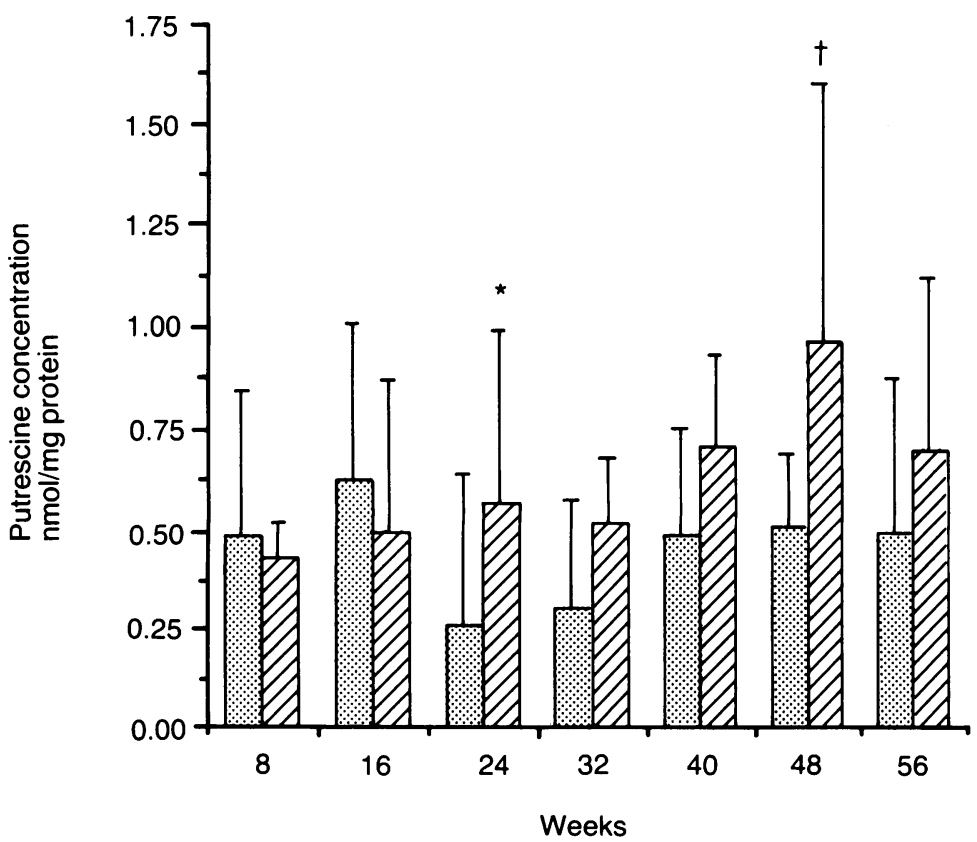

Figure 8: Putrescine concentration at various weeks after operation (mean $(S E M)) .{ }^{\star} p<0.05$; $t p<0 \cdot 01$. paper assessing the effects of chemically induced carcinogenesis which also showed a carcinoma yield of $15 \% .^{17}$

Many previous studies on the effects of surgery on the production of carcinomas in the rat gastric mucosa have used an inadequate control group. Most have used unoperated rats as controls. ${ }^{81819}$ Therefore the effects of surgery alone on the gastric mucosa have not been examined. We did not use vagotomy in the gastrojejunostomy group for two reasons. Firstly, a truncal vagotomy in a rat is associated with a high mortality, and secondly, we have previously reported that vagotomy does not increase the number of carcinomas found after a gastrojejunostomy in this model. ${ }^{20}$

Various features found in the gastrojejunostomy group are common to the control group and are related to surgical damage to the mucosa rather than duodenogastric reflux. These include foveolar hyperplasia, loss of specialised cells, glandular mucosal atrophy adjacent to the anastomosis, adenocystic glandular proliferation, downward glandular proliferation, and the finding of normal epithelium in deeper layers of the mucosa and pseudopyloric metaplasia. Adenocystic glandular proliferation was found in both groups and increased with time. In contrast, the incidence of normal epithelium in deeper layers decreased with time. This suggests that adenocystic glandular proliferation developed from normal epithelium in deeper layers.

Intestinal metaplasia was found only in gastrojejunostomy rats but did not increase with time. Only one animal had an area of intestinal metaplasia adjacent to high grade dysplasia. This confirms the findings of previous studies that in this model intestinal metaplasia is probably not an important premalignant change.

This study shows that carcinomas can be induced in the rat by surgery alone and that hyperplasia of the proliferative neck zone is associated with malignant change. The increase in sulphomucin producing cells, with lengthening of the neck zone, signifies an increase in the number of immature cells in the region immediately adjacent to the stoma. This change was rare in those animals undergoing gastrotomy.

At all time periods during the experiment ornithine decarboxylase activity was higher in the gastric mucosa of the gastrojejunostomy animals compared with the gastrotomy controls and this is consistent with the labelling index results. For two reasons we believe that this increase in ornithine decarboxylase activity is unlikely to have been due to bacterial contamination. Firstly, in previous work from our unit we have shown that there are only minor changes in the bacterial flora of gastric contents between gastrotomy rats and those subjected to duodenogastric reflux.' Secondly, ornithine decarboxylase activity was estimated at $\mathrm{pH} 7 \cdot 2-$ the $\mathrm{pH}$ at which mammalian ornithine decarboxylase activity is optimum. In contrast, the $\mathrm{pH}$ optimum for ornithine decarboxylase activity produced by Escherichia coli is $8 \cdot 1$, and for Lactobacillus $5 \cdot 8$. Therefore at the $\mathrm{pH}$ of our assay $(7 \cdot 2)$, bacterial ornithine decarboxylase activity would have been much reduced com- 


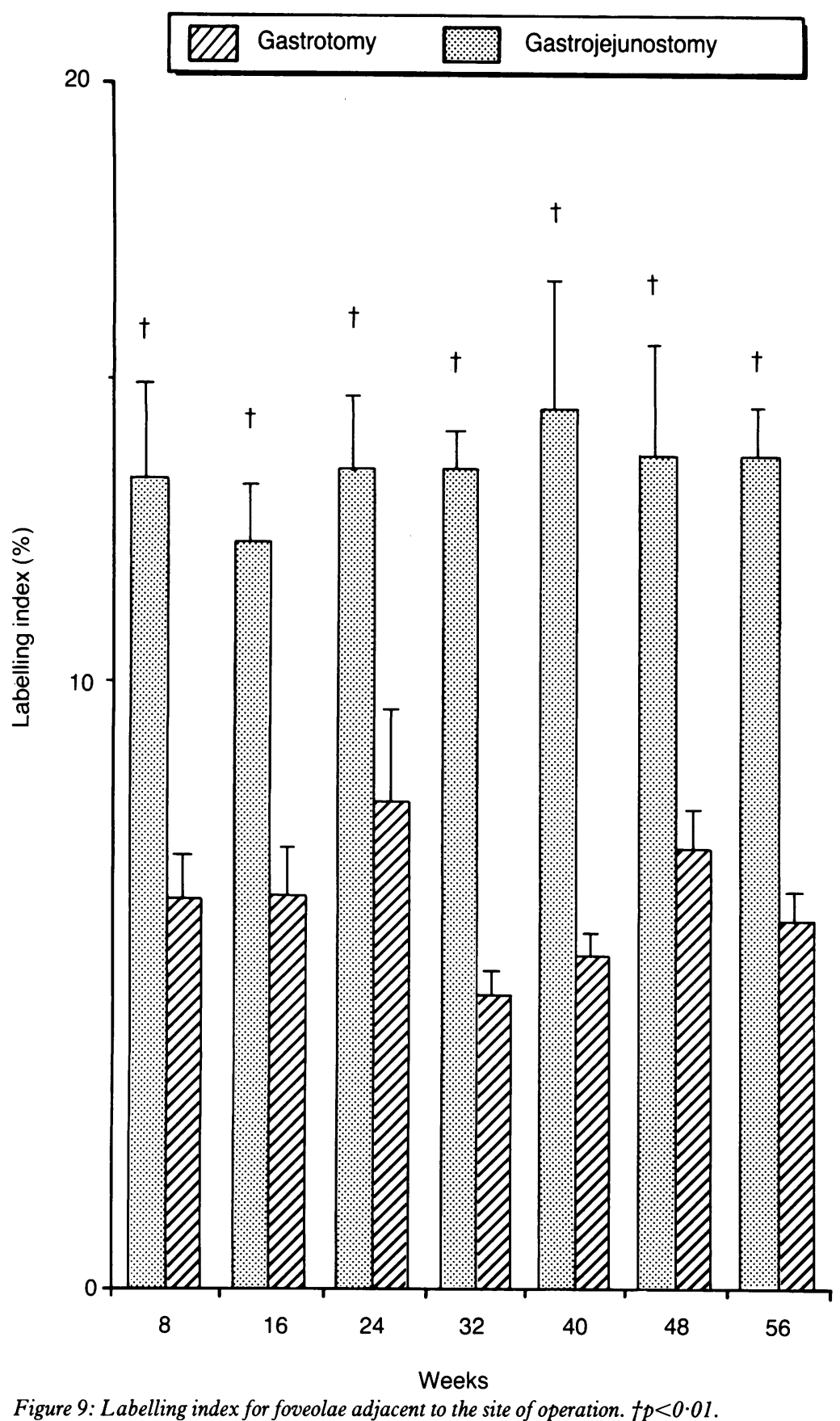

Figure 9: Labelling index for foveolae adjacent to the site of operation. $t_{p}<0.01$.

TABLE II Labelling index for histopathological lesions in gastrojejunostomy group

\begin{tabular}{lll}
\hline Histological lesion & No & Mean labelling index \\
\hline Carcinoma & 6 & $37 \cdot 6$ \\
High grade dysplasia & 1 & $32 \cdot 5$ \\
Low grade dysplasia & 6 & $16 \cdot 5$ \\
Polyps & 3 & $24 \cdot 7$ \\
\hline
\end{tabular}

$\star$ Two hyperplastic and one adenomatous polyps.

pared with mammalian activity, and in this way the two can be readily distinguished. ${ }^{21}$

The overall increase in ornithine decarboxylase activity of $36 \%$ in the gastrojejunostomy group compared with the control group was associated with an equivalent increase $(44 \%)$ in putrescine concentration. The fact that a similar pattern of results was seen at every time interval after surgery also suggests that the increase in putrescine concentration is indeed the result of the increased ornithine decarboxylase activity. There was, however, no increase in spermidine concentration. Increases in putrescine concentration are usually associated with increases in spermidine concentration, and both these polyamines are believed to be growth stimulating factors. Putrescine concentrations are controlled by at least five enzymes, however. Putrescine may be converted to spermidine, which in turn is converted to spermine. A reverse pathway exists (for the synthesis of spermidine from spermine, and for the conversion of spermidine to putrescine) but the extent to which this occurs is currently not known. ${ }^{22}$ Putrescine may also be oxidised by the enzyme diamine oxidase, to yield gamma-amino buteraldehyde and other derivatives. The concentrations of putrescine and spermidine therefore are related in a complex fashion, and further experiments need to be performed to elucidate their roles in this model.

In contrast to the increase in putrescine concentration, spermine concentrations were decreased in the gastrojejunostomy group when compared with gastrotomy controls. This finding also requires further study.

The results suggest that in the rat the gastric mucosa adjacent to a gastrojejunostomy has a higher rate of cell proliferation than that seen close to a gastrotomy scar. A correlation between increased cellular proliferation and carcinogenesis is well established. This study indicates that reflux of duodenal contents into the stomach induced by gastrojejunostomy promotes gastric mucosal hyperplasia. This increase in cell proliferation was apparent as early as eight weeks and persisted for 56 weeks after surgery. Our findings support the theory that hyperplasia precedes the development of carcinoma in this model.

This work was carried out as part of the requirement for the degree of MChir, Cambridge University.

1 Schafer LW, Larson DE, Melton LJ, et al. The risk of gastric carcinoma after surgical treatment for benign ulcer disease. N Engl F Med 1983; 309: 1210-3.

2 Sandler RS, Johnson MD, Holland KL. Risk of stomach cancer after gastric surgery for benign conditions: a case control study. Dis Dig Sci 1984; 29: 703-8.

3 Clark CG, Fresini A, Gledhill T. Cancer following gastric surgery. Br f Surg 1985; 72: 591-4.

4 Stalsberg $\mathbf{H}$, Taksdal S. Stomach cancer following surgery for benign conditions. Lancet 1971; ii: 1175-7.

5 Schrumpf E, Serck-Hansen A, Stadaas J, et al. Mucosal changes in the stump 20-25 years after partial gastrectomy. Lancet 1977; ii: 467-9.

6 Viste A, Bjornestad E, Opheim P, et al. Risk of carcinoma following gastric operations for benign disease. Lancet 1986; ii: $502-5$.

7 Caygill CPJ, Hill MJ, Kirkham JS, et al. Mortality from gastric cancer following gastric surgery for peptic ulcer. Lancet 1986; ii: 929-31.

8 Langhans $\mathrm{P}$, Heger R, Hohenstein J, et al. Operation sequel carcinoma of the stomach. Experimental studies of surgical techniques with or without resection. World f Sur 1981; 5 : 595-605.

9 Mason RC. Duodenogastric reflux in rat gastric carcinoma. Br7 Surg 1986; 73: 801-3.

10 Black O, Chang BK. Ornithine decarboxylase enzyme activity in human and hamster pancreatic tumor cell lines. Cancer Letts 1982; 17: 87-93.

11 Bauknecht T, Kleine W, Meerpohl HG. EGF receptors and ornithine decarboxylase: prognostic value in ovarian carcinomas. F Steroid Biochem 1983; 19 (suppl): 1189.

12 Filipe MI, Lake BD, eds. Histochemistry in pathology. Edinburgh: Churchill Livingstone, 1983: 310-3.

13 Rogers AW. Techniques of autoradiography. Amsterdam: Elsevier, 1979.

14 Luk GD, Baylin SB. Polyamines and intestinal growth increased polyamine biosynthesis after jejunectomy. $A m \mathcal{F}$ Physiol 1983; 245: G656-60.

15 Lowry OH, Rosebrough NJ, Farr AL, et al. Protein measurement with Folin phenol reagent. $\mathcal{F}$ Biol Chem 1951; 193: 265-73.

16 Seiler N, Knodgen B. High performance liquid chromatographic procedure for the simultaneous determination of the natural polyamines and their monoacetyl derivatives. natural polyamines and their
f Chromatogr 1980; 221: 227-35. 
17 Houghton PWJ, Mortensen NJMcC, Williamson RCN. Effect of duodenogastric reflux on gastric mucosal proliferation of duodenogastric reflux on gastric surgery. Br $\mathcal{F}$ Surg 1987; 74: 288-91.

18 Dahm K, Werner E, Eichen R, et al. Experimental cancer of the gastric stump. In: Herfarth Ch, Schlag P, eds. Gastric the gastric stump. In: Herfarth Ch, Schlag P, eds. Gastric

19 Schlake W, Nomura K. Histogenesis of carcinoma of the glandular stomach of the rat after BI resection. In: Grundmann E, Kirsten WH, eds. Current topics in pathology. Carcinogenesis. Vol 68. Berlin: Springer-Verlag, 1979.
20 Taylor PR, Hanley DC, Filipe MI, Mason RC. What factor is responsible for malignant change in the rat stomach following truncal vagotomy and gastroenterostomy? In: Reed PI, Hill MJ, eds. Gastric carcinogenesis. Oxford: Excerpta Medica, 1988: 29 .
.

21 Kaye AM. Ornithine decarboxylase. Purification and properties of ornithine decarboxylase. Cell Biochem Funct 1984; 2 . 2-6.

22 Pegg AE. Recent advances in the biochemistry of polyamines in eukaryotes. Biochem $\mathcal{F}$ 1986; 234: 249-62. 